\title{
Delayed neutron yields and spectra from photofission of actinides: data and calculations with Bremsstrahlung photons below $20 \mathrm{MeV}$
}

\author{
D. Doré ${ }^{1}$, J.-C. David ${ }^{1}$, V. Macary ${ }^{1}$, J.-M. Laborie ${ }^{2}$, X. Ledoux ${ }^{2}$, D. Ridikas ${ }^{1}$, and A. Vanlauwe ${ }^{2}$ \\ DSM/DAPNIA/SPhN, CEA/Saclay, 91191 Gif-sur-Yvette, France \\ 2 DAM/DPTA/SPN, CEA/DIF, 91680 Bruyères-le-Châtel, France
}

\begin{abstract}
Photonuclear reactions, especially photofission delayed neutron emission, play an important role for applications involving nuclear material detection. In order to provide good quality data for evaluations and data libraries, an experimental program of delayed neutron measurements has been undertaken for actinides with Bremsstrahlung energy from 12 up to $20 \mathrm{MeV}$. In this paper, we report on delayed neutron yields and time spectra characteristics from the photofission of ${ }^{238} \mathrm{U}$ and ${ }^{235} \mathrm{U}$ for several endpoint energies between 12 and $18.5 \mathrm{MeV}$. The associated modelling efforts will be described including some calculations compared to experimental data.
\end{abstract}

\section{Introduction}

A renewed interest in photonuclear reactions was stimulated by applications as radioactive ion beam production, shielding of electron accelerators, production of intense neutron beams, etc. Today, a particular attention is paid to the nondestructive characterization of waste barrels and detection of nuclear materials, both based on photofission process and the associated delayed neutron (DN) emission. Need of accurate and complete data for DN yields and time characteristics of actinides was the motivation for the experimental campaign, started in 2004. The experimental effort is supported by a complete modelling of the process.

The contributions of around $200 \mathrm{DN}$ precursors are generally lumped into 6 groups according to their half-lives $t_{1 / 2}$ going from $50 \mathrm{~ms}$ to $55 \mathrm{~s}$. For each group $i$, two parameters are determined: the DN contribution $a_{i}$ and the mean decay constant of the group $\lambda_{i}\left(\ln 2 / t_{1 / 2}\right)$ leading to a simplified description of the $\mathrm{DN}$ decay curve through the relation

$$
Y_{D N}(t)=\sum_{i=1}^{6} a_{i} e^{-\lambda_{i} t}\left(1-e^{-\lambda_{i} T_{i r r}}\right)
$$

where $\mathrm{T}_{\text {irr }}$ is the irradiation period. The sum of all $a_{i}$ is called $v_{\mathrm{d}}$ and is characteristic for a fissionable nucleus. For photo-fission experiments, $a_{i}, \lambda_{\mathrm{i}}$ and $v_{\mathrm{d}}$ are extracted from time dependent DN measurements. $\mathrm{T}_{\text {irr }}$ and $\mathrm{T}_{\mathrm{dec}}$ (decay time) are varied to enhance alternatively each group contribution with respect to others. From 2004 to 2006, four experiments have been performed with different actinides $\left({ }^{238} \mathrm{U},{ }^{232} \mathrm{Th}\right.$, ${ }^{235} \mathrm{U}$ ) at different electron energies (from 12 to $18.5 \mathrm{MeV}$ ) [1-3] and with three different experimental setups. In this paper, we describe 2006's experiments and show preliminary data for absolute yield determination and group parameter extraction. Modelling work [3,4] is also briefly presented and results compared to experimental ones. Finally, importance of precise measurements for group parameters is emphasized with an example aiming at the isotopic identification of a mixed sample.

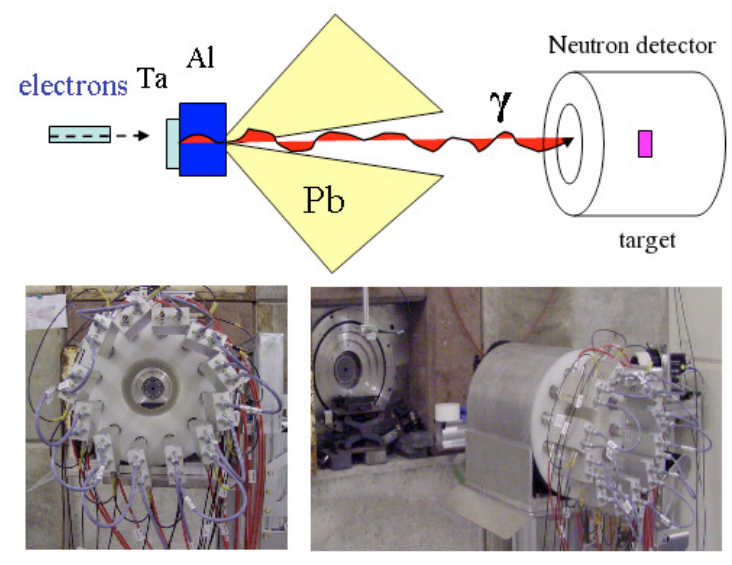

Fig. 1. Top: experimental setup for DN measurements. Bottom: photos of the $\mathrm{DN}$ detector.

\section{Experimental setup and method}

The ELSA electron accelerator of CEA/DIF/DPTA was used to provide end-point Bremsstrahlung photons with energies from 12 to $19 \mathrm{MeV}$. A simplified scheme of the experiment is shown on figure 1 . The electron beam, with intensity around $1 \mu \mathrm{A}$ and energy resolution $\sim 100 \mathrm{keV}$, impinges on the $2 \mathrm{~mm}$ thick Ta target. The Al cylinder stops the residual electrons. $\mathrm{A} \mathrm{Pb}$ collimator placed at 0 degree defines the photon beam opening angle. At $2.34 \mathrm{~m}$ (target position), the photon beam spot has a diameter of $6.7 \mathrm{~cm}$ and the beam intensity is around $10^{8} \mathrm{\gamma} / \mathrm{cm}^{2}$.

First experiments were performed with a basic neutron detector (low efficiency) and important target masses (400 g). Since 2006, due to the low mass of actinide available and limited beam intensity (due to radioprotection constraints), a new detector has been designed and constructed (fig. 1). Twelve ${ }^{3} \mathrm{He}$ gas counters were uniformly distributed inside a $\mathrm{CH}_{2}$ ring of $37 \mathrm{~cm}$ long with inner and outer radius of 6 and $16 \mathrm{~cm}$, respectively. The ensemble was wrapped in a $1 \mathrm{~mm}$ thick $\mathrm{Cd}$ foil to avoid low energy background neutrons to 


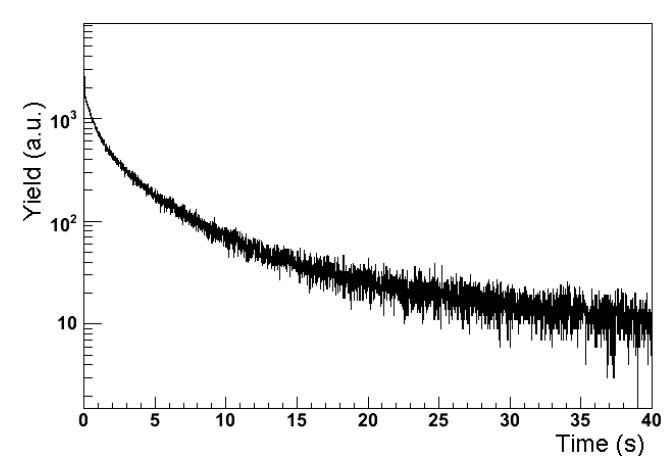

Fig. 2. DN time dependent yield for ${ }^{238} \mathrm{U}$ after 70 cycles with $\mathrm{T}_{\text {irr }}=100 \mu \mathrm{s}$.

be detected. $\mathrm{CH}_{2}$ thickness has been optimized to obtain a constant efficiency over the expected DN energy range $(0.1$ to $1.0 \mathrm{MeV}$ ). Within this configuration an efficiency of $\sim 20 \%$ is obtained, a gain of a factor 10 compared to our previous DN detectors.

The experimental method consists of repetitive cycles of irradiation and decay periods. To extract group parameter three different combinations of irradiation-decay times are performed: $300 \mathrm{~s}-300 \mathrm{~s} ; 10 \mathrm{~s}-100 \mathrm{~s}$ and $100 \mu \mathrm{s}-40 \mathrm{~s}$. For absolute DN yield measurements, the short irradiation is used.

\subsection{Absolute DN yields}

In November 2006, the whole range of electron energy between 12 and $18.5 \mathrm{MeV}$ has been covered to measure absolute DN yields for ${ }^{238} \mathrm{U}(400 \mathrm{~g})$ and ${ }^{232} \mathrm{Th}(300 \mathrm{~g})$. For ${ }^{235} \mathrm{U}(11 \mathrm{~g})$, only few points have been studied. A typical decay spectrum of ${ }^{238} \mathrm{U}$ DN yields, obtained after 70 repetition cycles with $100 \mu \mathrm{s}-40 \mathrm{~s}$ irradiation-decay sequences, is presented in figure 2.

For a $\mathrm{T}_{\text {irr }} \ll$ shortest $\mathrm{T}_{1 / 2}^{\mathrm{i}}$, the absolute yield $\left(v_{\mathrm{d}}\right)$ is the ratio of the emitted $\mathrm{DN}$ over the number of fissions. The number of $\mathrm{DN}$ is the integral of the curve on figure 2 corrected for background, detector efficiency and contribution of (n,fiss) reactions. The number of fissions (induced by photon and neutrons) is evaluated using the MCNPX transport code. The total beam charge was recorded during the irradiations. The exact geometrical description of the set-up (converter; collimator and sample) is taken into account in the simulation. Figures 3 and 4 show preliminary results of total DN yields (full symbols) for the ${ }^{238} \mathrm{U}$ and ${ }^{235} \mathrm{U}$ targets. Error bars are only statistical. The systematic uncertainty due to the electron charge measurements is difficult to evaluate and has not yet been taken into account. Previous results of Nikotin et al. [5] at $15 \mathrm{MeV}$ are also shown in figures 3 and 4 (open circles). As can be seen from these comparisons, our data are coherent with Nikotin's data giving the uncertainties which are reduced. Some other experimental data are also plotted on figures 3 and $4[6,7]$. Here again, within the error bars, all data are consistent with our preliminary results. We note separately that in the case of the ${ }^{235} \mathrm{U}$ target, containing $7 \%$ of ${ }^{238} \mathrm{U}$, the contribution of ${ }^{238} \mathrm{U}(\gamma, \mathrm{f})$ had to be subtracted. This will be done after complete analysis of the present ${ }^{238} \mathrm{U}$ data.

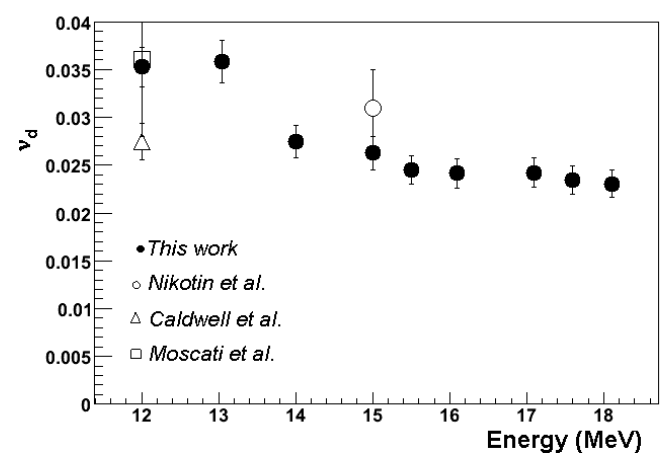

Fig. 3. Absolute DN yields for ${ }^{238} \mathrm{U}$ as a function of electron energy.

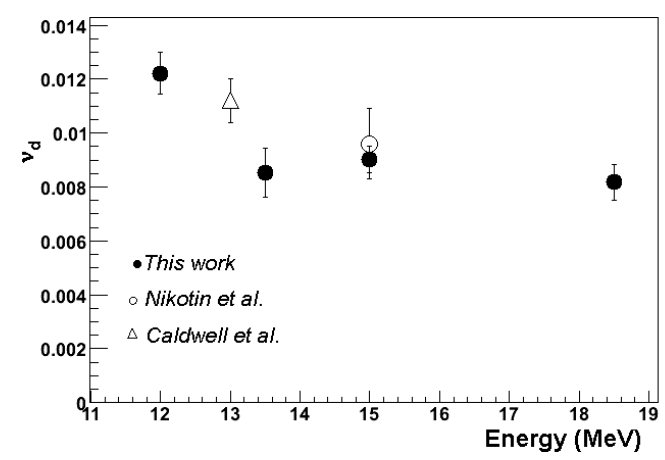

Fig. 4. Same as Figure 3 but for ${ }^{235} \mathrm{U}$.

The behaviour of $v_{\mathrm{d}}$ as a function of the electron energy is also of our primary interest. Trends are similar for ${ }^{235} \mathrm{U}$ and ${ }^{238} \mathrm{U}$ with a decrease of $v_{\mathrm{d}}$ around $13 \mathrm{MeV}$. This decrease is generally interpreted as the opening of the $2^{\text {nd }}$ chance fission channel $\left(\mathrm{E}_{\mathrm{thr}} \sim 12 \mathrm{MeV}\right)$ but more precise interpretation has still to be studied.

\subsection{Group parameters}

Available data for ${ }^{238} \mathrm{U}$ DN six group parameters show important discrepancies. For example, Kull et al. [8] have reported an important difference for contribution of groups 4 and 6 in respect to Nikotin's data. It can be seen in figure 5, where there is a factor of two between Kull's and Nikotin's data for these groups. Kull et al. have attributed these discrepancies to the opening of the $2^{\text {nd }}$ chance fission around $15 \mathrm{MeV}$.

We have determined group parameters for ${ }^{238} \mathrm{U}$ at 15 and $18 \mathrm{MeV}$ previously. As mentioned earlier, in order to extract $a_{i}$ and $\lambda_{\mathrm{i}}$ for all groups, three types of irradiation are used. First, for the 300-300 s combination, the parameters for groups 1 and 2 have been evaluated. Then, these parameters have been fixed and from the second type of irradiation (10$100 \mathrm{~s}$ ), parameters of groups 3 and 4 have been extracted. Finally, using the short irradiation spectrum, $5^{\text {th }}$ and $6^{\text {th }}$ group parameters have been determined. Results shown on figure 5 for $15 \mathrm{MeV}$ (black squares) and $18 \mathrm{MeV}$ (black points) have confirmed Kull's data. It seems that there is no evolution of $a_{i}$ between 8 and $18 \mathrm{MeV}$. This observation concerning group 


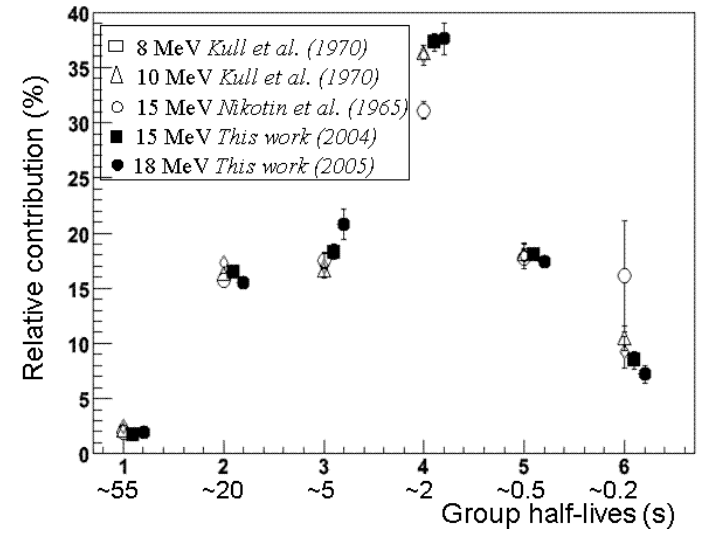

Fig. 5. Relative DN group contributions for photofission of ${ }^{238} \mathrm{U}$.

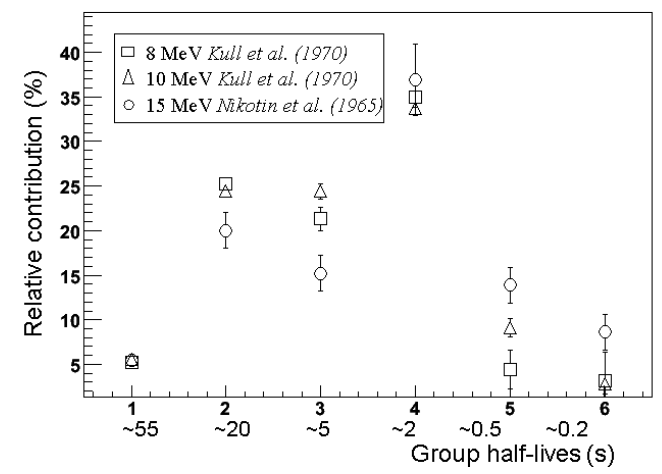

Fig. 6. Same as figure 5 but for ${ }^{235} \mathrm{U}$.

5 and 6 is particularly important for applications and will be discussed in section 3 .

For ${ }^{235} \mathrm{U}$ (fig. 6), Kull's data at 8 and $10 \mathrm{MeV}$ (open squares and triangles) are rather close except for group 3 and 5. However, discrepancies with Nikotin's data appear for almost all groups. Our analysis for ${ }^{235} \mathrm{U}$ is still in progress but already seems to indicate that here again Nikotin's data overestimates the $6^{\text {th }}$ group contribution.

\section{Modeling}

The modeling work is an important step in order to provide group parameter for all actinides. The whole process has been taken into account in the developed calculations. First of all, the Bremsstrahlung photon spectrum inside the actinide sample is calculated with the MCNPX code (ref. [9]) taking into account the exact experimental set-up. In the $\gamma$-excitation of nucleus we considered only the Giant Dipole Resonance contribution, where an empirical systematic based on the sum of two Lorentzian distributions seems to describe this process properly below $20 \mathrm{MeV}$.

The de-excitation of the nucleus is treated by the ABLA model developed at GSI [10] known to give good results in the case of high energy spallation reactions. Competition between particle emission and fission is treated by a statistical

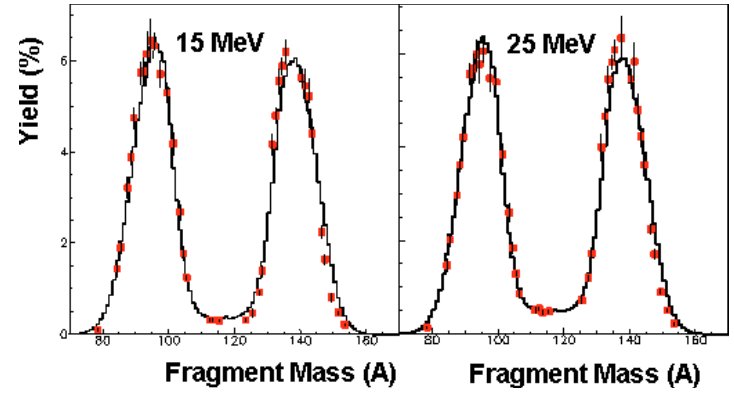

Fig. 7. Predicted and measured mass distributions for photofission of ${ }^{235} \mathrm{U}$ at 15 and $25 \mathrm{MeV}$.

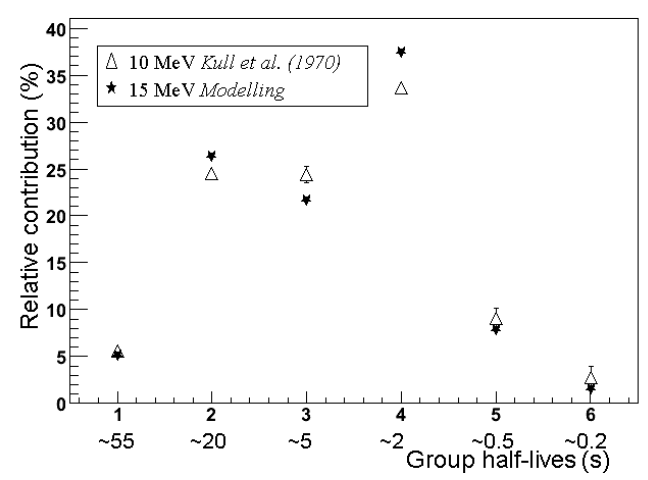

Fig. 8. Relative DN group contributions for photofission of ${ }^{235} \mathrm{U}$.

model. In other words, the complete code provides neutron emission, fission cross section and also fission yields. Note that the multi-chance fissions are taken into account as well. At this step, some experimental fission mass distributions are available to be compared to predicted ones. In figure $7,{ }^{235} \mathrm{U}$ mass yields show a good agreement with Jacobs and Thierens data [11-13]. Similar quality is also obtained for ${ }^{238} \mathrm{U},{ }^{237} \mathrm{~Np}$ and ${ }^{239} \mathrm{Pu}$. Isotopic yields, closely related to our observable, i.e., DN yields, are presented in [3].

To treat the calculation of DNs, the independent fission yields predicted by ABLA are transferred into cumulative yields using the CINDER'90 transmutation-decay code [14]. The DN precursors are identified and selected according to the nuclear data tables. Using tabulated DN emission probabilities and half-lives, all precursors are merged into six DN groups according to their half-lives. In this way the model provides a full set of DN parameters (total yield $v_{\mathrm{d}}$, six group yields $a_{i}$ and averaged group time constants $\lambda_{\mathrm{i}}$ ).

Calculated group yields (stars) for ${ }^{235} \mathrm{U}$ are compared to experimental data (triangles) in figure 8 . The general agreement is good and especially for group 5 and 6, which have an important role for applications such the one described below.

Discrepancies for groups 3 and 4 seem to be systematic since it has also been observed for ${ }^{238} \mathrm{U}$ and ${ }^{232} \mathrm{Th}$. This shift has to be investigated.

\section{Isotopic identification}

Irradiation of actinide mixtures was also performed in order to test the possibility to determine the composition and the 


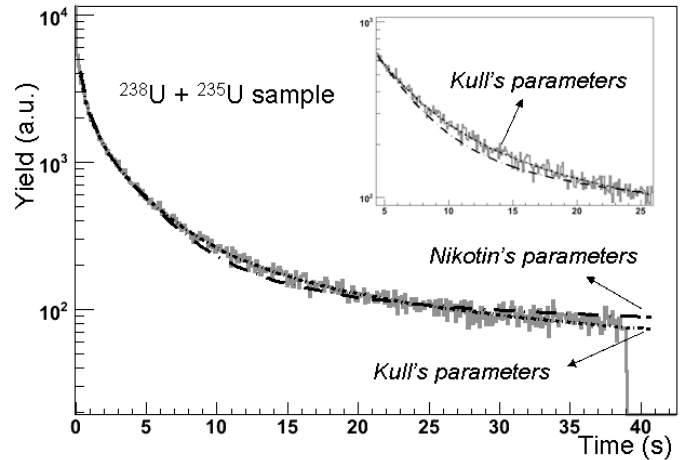

Fig. 9. DN time dependent yield for a mixture of ${ }^{235} U$ and ${ }^{238} U$ at $15 \mathrm{MeV}$.

mass of unknown composite samples. Precise measurements for the $5^{\text {th }}$ and $6^{\text {th }}$ group parameters are very important for such identification since short irradiation is used, as it would be probably the case for an eventual application. In figure 9, DN time dependent yield of a sample composed of $11 \mathrm{~g}$ of ${ }^{235} \mathrm{U}$ and $10 \mathrm{~g}$ of ${ }^{238} \mathrm{U}$ is shown. Hundred cycles of $\mathrm{T}_{\text {irr }}=$ $100 \mu \mathrm{s}-\mathrm{T}_{\mathrm{dec}}=40 \mathrm{~s}$ were performed. Our analysis shows that using only one baseline (one actinide) it is impossible to reproduce the experimental curve. Taking into account both actinides, the experimental time dependence is obtained by fitting only the background and mass of both actinides. Using parameters of Nikotin for ${ }^{238} \mathrm{U}$ and ${ }^{235} \mathrm{U}$, the fit (dashed line) does not reproduce the experimental curve since the ${ }^{238} \mathrm{U}$ contribution is set to a negative value. Fortunately, with Kull's parameter (dotted line) data are well reproduced. This result argues in favour of Kull's data analysis both for ${ }^{235} \mathrm{U}$ and ${ }^{238} \mathrm{U}$. The next step, still in progress, will be the mass evaluations.

\section{Conclusions}

An experimental campaign launched in 2004 for actinide DN measurements from photofission below $20 \mathrm{MeV}$ is giving interesting results. Absolute DN yields for ${ }^{238} \mathrm{U},{ }^{232} \mathrm{Th}$ and ${ }^{235} \mathrm{U}$ have been obtained for several Bremsstrahlung endpoint energies. Data are precise and energy dependence becomes clearer. Our experimental values will be included in the evaluated files obtained by E. Dupont et al. (in this conference) and to be provided to the JEFF community. For group parameters, the situation for the $6^{\text {th }}$ group of ${ }^{238} \mathrm{U}$ and ${ }^{232} \mathrm{Th}$ was clarified and old values at $15 \mathrm{MeV}$ were corrected. Preliminary results for

${ }^{235} \mathrm{U}$ seem to indicate the same feature. These new parameters allow determining the actinide content of a mixed actinide sample. Many mixtures with different proportions have been studied and analysis is in progress. Modelling has also been improved and isotopic yield as well of mass distributions are rather well reproduced by the calculations. Systematic differences still have to be explained.

In 2007 and $2008,{ }^{237} \mathrm{~Np}$ and ${ }^{239} \mathrm{Pu}$ targets will be available, and therefore absolute yields and group parameters will be measured for some Bremsstrahlung endpoint energies. These measurements will be done in parallel with detection of photofission delayed photons (DP). These experiments aim providing the DP/DN ratios in the frame of the INPHO project presently in progress at CEA.

Authors thank the staff of the ELSA accelerator for their support and fine working conditions during the experiments.

\section{References}

1. M.-L. Giacri-Mauborgne, Ph.D. thesis, University of Caen, 2005; Report DAPNIA-05-14-T.

2. D. Doré et al., EPS J. Phys.: Conf. Ser. 41, 241 (2006).

3. A. Van Lauwe et al., Proc. of Int. Conf. on Nuclear Reaction Mechanisms, Varenna, Italy, 12-16 June, 2006.

4. J.-C. David et al., Proc. of the Int. Conf. on Nuclear Data for Science and Technology, ND2004, Santa Fe, New Mexico, USA, 26 Sept. -1 Oct. 2004.

5. O.P. Nikotin, K.A. Petrzhak, Atomnaya Energya 20, 268 (1965).

6. J.T. Caldwell et al., Nucl. Sci. Eng. 56, 179 (1965).

7. G. Moscati et al., Phys. Rev. 126 (1961)

8. L.A. Kull et al., Nucl. Sci. Eng. 39, 163 (1970).

9. J. Hendricks et al., MCNPX extensions: version 2.5.0, LA-UR05-2675, LANL, USA, April 2005.

10. A.R. Junghans et al., Nucl. Phys. A 629, 635 (1998); J. Benlliure et al., Nucl. Phys. A 628, 458 (1997).

11. E. Jacobs et al., Phys. Rev. C 19, 422 (1979).

12. H. Thierens et al., Phys. Rev. C 14, 1058 (1976).

13. E. Jacobs et al., Phys. Rev. C 21, 237 (1980).

14. W.B. Wilson, T.R. England, K.A. Van Riper, Status of CINDER'90 Codes and Data, Los Alamos National Laboratory, Report LA-UR-99-361, 1999. 